\title{
Comments: "Low triiodothyronine syndrome is associated with platelet function in patients with nephrotic syndrome"
}

1. Harvard Medical School, Department of Neurosurgery, Brigham and Women's Hospital, Boston, Massachusetts, USA 2. Neuroscience Institute, Lithuanian University of Health Sciences, Kaunas, Lithuania E-mail: abunevicius@bwh.harvard.edu

Low triiodothyronine (TL3) syndrome is a common complication of critical illnesses and has been documented as an independent predictor of greater severity and unfavorable prognosis ${ }^{1}$. Impaired thyroxin to T3 5'-deiodination in peripheral tissues and the brain was implicated as the major mechanism underlying the development of LT3 syndrome. T3 is the most potent of the two thyroid gland hormones, and reduced concentrations of T3 can significantly impair cell metabolism. Mechanisms underlying the association of LT3 syndrome with unfavorable outcomes remain largely unknown, and the treatment for LT3 syndrome has not been shown to improve outcomes of critically ill patients with reduced T3 concentrations. Furthermore, it is also argued that LT3 syndrome can be an adaptive response to conserve energy in severely ill patients.

A study by Wu et al. ${ }^{2}$ on patients with nephrotic syndrome suggests that LT3 syndrome is associated with a pro-coagulative and pro-thrombotic state. Specifically, they found that nephrotic disease patients with LT3 syndrome, compared with patients with no LT3 syndrome, have prolonged activated partial thrombin time (APTT), greater platelet activation (CD63 measured with flow cytometry), and adenosine diphosphate-induced platelet aggregation rate (PAgTAA), which is indicative of a pro-coagulative and pro-thrombotic state.

Greater platelet aggregation and pro-coagulative state associated with LT3 syndrome can be an important and previously undescribed mechanism under- lying the well-documented, strong and independent association of LT3 syndrome with greater disease severity, mortality, and unfavorable prognosis of patients with vascular disorders, including stroke ${ }^{3}$ and myocardial infarction ${ }^{4}$. These findings suggest that patients with acute vascular disorders (MI and stroke) who develop LT3 syndrome are at high risk of developing a pro-coagulative and pro-thrombotic state that can subsequentially cause propagation of blood clots within the affected artery, resulting in recruitment of branching vessels and, consequentially, in greater volume of infarcted myocardium or brain tissue. Greater end-organ damage subsequently correlates with worse clinical status, limited efficacy of commonly used therapeutic interventions, and worse overall outcomes. At subacute disease phases, thromboembolic complications (such as deep vein thrombosis and pulmonary embolism) can be serious, potentially fatal, and sometimes occur despite pharmacologic and mechanical preventive measures. Hence, it is possible that untreated LT3 syndrome can place critically ill patients at high risk of thromboembolic complications at sub-acute disease phases and, thus, result in devastating outcomes, impaired recovery, and limited participation in rehabilitation.

Ischemic stroke patients with LT3 syndrome also have worse cognitive outcomes when compared to patients with no LT3 syndrome, an association independent from stroke severity and patient age ${ }^{5}$. It can be hypothesized that a pro-coagulative and pro-thrombotic state associated with LT3 syndrome 
can predispose microvascular circulation impairment in brain areas responsible to cognitive functioning, such as the limbic system, and remote from the stroke territory.

Further studies exploring pro-coagulative and pro-thrombotic state as a possible underlying complication of LT3 syndrome in patients with acute cerebrovascular and cardiovascular disorders are strongly encouraged as they could provide a potentially actionable target for treatment with safe and widely used hormone replacement therapy ${ }^{5}$.

\section{REFERENCES}

1. Fliers $E$, Bianco $A C$, Langouche $L$, Boelen $A$. Thyroid function in critically ill patients. Lancet Diabetes Endocrinol. 2015;3(10):816-25.

2. Wu J, Guo N, Chen X, Xing C. Low triiodothyronine syndrome is associated with platelet function in patients with nephrotic syndrome. Rev Assoc Med Bras. 2019; 65(7)988-992.

3. Lamba N, Liu C, Zaidi H, Broekman MLD, Simjian T, Shi C, et al. A prognostic role for low tri-iodothyronine syndrome in acute stroke patients: a systematic review and meta-analysis. Clin Neurol Neurosurg. 2018;169:55-63.

4. Wang B, Liu S, Li L, Yao Q, Song R, Shao X, et al. Non-thyroidal illness syndrome in patients with cardiovascular diseases: a systematic review and meta-analysis. Int J Cardiol. 2017;226:1-10

5. Bunevicius A, Kazlauskas H, Raskauskiene N, Janusonis V, Bunevicius R. Ischemic stroke functional outcomes are independently associated with C-reactive protein concentrations and cognitive outcomes with triiodothyronine concentrations: a pilot study. Endocrine. 2014;45(2):213-20. 